Characterization of iron compounds in tumour tissue from temporal lobe epilepsy patients using low temperature magnetic methods

\author{
Journal Article \\ Author(s): \\ Brem, Franziska; Hirt, Ann Marie (D); Simon, Christian; Wieser, Heinz-Gregor; Dobson, Jon \\ Publication date: \\ 2005-04 \\ Permanent link: \\ https://doi.org/10.3929/ethz-b-000033269 \\ Rights / license: \\ In Copyright - Non-Commercial Use Permitted \\ Originally published in: \\ BioMetals 18(2), https://doi.org/10.1007/s10534-004-6253-y
}




\title{
Characterization of iron compounds in tumour tissue from temporal lobe epilepsy patients using low temperature magnetic methods
}

\author{
Franziska Brem ${ }^{1, *}$, Ann M. Hirt ${ }^{1}$, Christian Simon ${ }^{2}$, Heinz-Gregor Wieser ${ }^{2}$ \& Jon \\ Dobson $^{3}$ \\ ${ }^{1}$ ETH-Hönggerberg, Institute of Geophysics, CH-8093 Zurich, Switzerland; ${ }^{2}$ University Hospital Zurich, \\ Neurology/EEG, CH-8091 Zurich, Switzerland; ${ }^{3}$ Keele University, Institute for Science \& Technology in \\ Medicine, Stoke-on-Trent, ST4 7QB, UK; *Author for correspondence (Tel.: +411-633-2626; Fax: \\ +411-633-1065; E-mail: brem@mag.ig.erdw.ethz.ch)
}

Received 25 August 2004; accepted 15 December 2004; Published online: March 2005

Key words: biogenic magnetite, brain ferritin, low temperature magnetic measurements, mesial temporal lobe epilepsy (MTLE), tumour tissue

\begin{abstract}
Excess iron accumulation in the brain has been shown to be related to a variety of neurodegenerative diseases. However, identification and characterization of iron compounds in human tissue is difficult because concentrations are very low. For the first time, a combination of low temperature magnetic methods was used to characterize iron compounds in tumour tissue from patients with mesial temporal lobe epilepsy (MTLE). Induced magnetization as a function of temperature was measured between 2 and $140 \mathrm{~K}$ after cooling in zero-field and after cooling in a $50 \mathrm{mT}$ field. These curves reveal an average blocking temperature for ferritin of $10 \mathrm{~K}$ and an anomaly due to magnetite at $48 \mathrm{~K}$. Hysteresis measurements at $5 \mathrm{~K}$ show a high coercivity phase that is unsaturated at $7 \mathrm{~T}$, which is typical for ferritin. Magnetite concentration was determined from the saturation remanent magnetization at $77 \mathrm{~K}$. Hysteresis measurements at various temperatures were used to examine the magnetic blocking of magnetite and ferritin. Our results demonstrate that low temperature magnetic measurements provide a useful and sensitive tool for the characterisation of magnetic iron compounds in human tissue.
\end{abstract}

\section{Introduction}

In the brain, iron plays an important role in normal neurological functions such as neurotransmitter synthesis and myelination. It is an essential component of physiological processes, such as oxygen and electron transport, but can also be toxic, acting as a catalyst for the production of free radicals. Many neurodegenerative diseases, such as Alzheimer's and Parkinson's disease, are associated with a disruption of iron metabolism (Beard et al. 1993). Iron-induced epilepsy and electrophysiological responses are described in several studies (Ueda et al. 1998; Ueda \& Willmore 2000) and iron overload may even lead to a predisposition to epilepsy (Ikeda 2001).
The primary iron storage system in most living organisms is in the form of nanoparticles of ferrihydrite $\left(5 \mathrm{Fe}_{2} \mathrm{O}_{3} \times 9 \mathrm{H}_{2} \mathrm{O}\right)$ encapsulated by a spherical protein shell. This iron storage protein, ferritin, maintains iron in an available soluble, non-toxic form. Each protein shell of ferritin consists of 24 polypeptide subunits forming an approximately spherical cage of $12 \mathrm{~nm}$ in diameter. Potentially toxic ferrous iron is sequestered by the shell and oxidized in the core. The internal cavity has a diameter of approximately $8 \mathrm{~nm}$ and is occupied by the ferrihydrite core which undergoes antiferromagnetic ordering at low temperature and has a net magnetic moment arising from frustrated surface spins due to its small size (Massover 1993; Harrison \& Arosio 1996; 
Chasteen \& Harrison 1999; Seehra et al. 2000). Many low temperature magnetometry studies have been performed on horse spleen ferritin (MohieEldin et al. 1994; Makhlouf et al. 1997; Luis et al. 1999; Gilles et al. 2002) and Dubiel et al. (1999) identified ferritin in globus pallidus tissue from the human brain. These authors found that ferritin in the brain had a smaller average core diameter and blocking temperature than ferritin in horse spleen.

Biogenic magnetite is another iron compound that has been identified in human brain tissue (Kirschvink et al. 1992; Dobson \& Grassi 1996; Schultheiss-Grassi \& Dobson 1999; Hautot et al. 2003) and in tumour tissue (Kobayashi 1997). Magnetite $\left(\mathrm{Fe}_{3} \mathrm{O}_{4}\right)$ is a ferrimagnetic iron oxide with an inverse spinel structure. It contains alternating sublattices of $\mathrm{Fe}^{2+}$ and $\mathrm{Fe}^{3+}$ ions, which are antiferromagnetically coupled. The alternation of the two sublattices with unequal numbers of unpaired electron spins leads to magnetite's strong ferrimagnetic magnetization. There is preliminary evidence that biogenic magnetite is associated with Alzheimer's disease and some studies have suggested that it may be responsible for the triggering of epileptiform activity (Fuller et al. 1995) and the production of free radicals (Schafer et al. 2000). Though the source of biogenic magnetite is still unknown, it has been suggested that ferritin may be a precursor for magnetite. If the ferritin protein becomes overloaded or if there is a malfunction in the iron transport channels, a mechanism for $\mathrm{Fe}^{2+}$ oxidation is lost (Quintana et al. 2000; Dobson 2001; Zhao et al. 2003).

Magnetometry methods, which were developed for investigations of magnetic materials, including sediments and rocks, are effective at detecting small concentrations of magnetic iron compounds within diamagnetic and paramagnetic matrices (Maher 1999; Evans 2003). These methods can provide proxy information on the iron compounds that are present in addition to their concentration, and in the case of magnetite, its particle size. Few studies have detected ferritin in the human brain with magnetic methods. The aim of this study is to identify ferritin and biogenic magnetite in brain tumour tissue from mesial temporal lobe epilepsy (MTLE) patients and to determine which magnetic methods are most suitable for the characterisation of these iron oxides in brain tissue.

\section{Material and methods}

Two brain tissue samples, GH (gemistocytic astrocytoma, WHO grading II) and NU (ganglioglioma, WHO grading I), were resected from patients with MTLE and immediately frozen at $-80{ }^{\circ} \mathrm{C}$. Four types of magnetic measurements were made: (1) acquisition of isothermal remanent magnetization (IRM); (2) thermal demagnetization of low temperature IRM; (3) measurement of induced magnetization (DC susceptibility) as a function of temperature; (4) induced magnetization as a function of field (hysteresis curves). All sample holders, vials, glass ware and surgical instruments used for the sample preparation were soaked in $\mathrm{HCl}$ for at least $24 \mathrm{~h}$ and rinsed with distilled water to prevent contamination. Precautions were taken to avoid contaminations, as described in earlier studies (Dobson \& Grassi 1996), and all samples were weighed prior to measuring.

Acquisition of IRM is useful in identifying ordered, blocked magnetic phases in a material because the measured remanent magnetization is not affected by diamagnetic or paramagnetic materials in the sample, such as tissue and heme iron. For IRM experiments frozen tissue samples were placed in a pre-cooled teflon holder and put into liquid nitrogen. The IRM measurements were done on a 3-axis $2 \mathrm{G}$ Enterprises Superconducting Quantum Interference Device (SQUID) with a sensitivity level of $10 \times 10^{-12} \mathrm{Am}^{2}$. First, the empty teflon holder was measured at $77 \mathrm{~K}$ and then the tissue was placed in the holder and measured in the same manner. The IRM was acquired in an ASC Scientific Pulse Magnetizer Model IM$10-30$, where the sample was exposed to a pulsed DC field. First, a pulse of 1 Tesla was applied to the sample and the magnetization was measured. Then, the sample was turned $180^{\circ}$ and fields from $10 \mathrm{mT}$ to $1 \mathrm{~T}$ were given in the opposite direction. After each pulse, the remanent magnetization in the tissue was measured after $120 \mathrm{~s}$. Afterwards, the tissue was removed and the sample holder was demagnetized in a $150 \mathrm{mT}$ AC field, to remove the acquired IRM. The procedure was repeated for the empty teflon sample holder so that the signal of the holder could then be subtracted from the total IRM signal of the holder and sample.

Thermal demagnetization of low temperature IRM was made on tissue samples that were freeze-dried to remove water. The freeze-dried 
tissue was pressed into a cylindrical pellet within a diamagnetic straw using teflon pistons made for this purpose. Thermal demagnetization of low temperature IRM was measured with a Quantum Design Magnetic Property Measurement System (MPMS) SQUID Magnetometer at the University of Bremen. The samples were initially cooled down to $2 \mathrm{~K}$ either in the absence of a magnetic field (zero-field cooled, ZFC) or in the presence of a strong $5 \mathrm{~T}$ field (field cooled, FC). At $2 \mathrm{~K}$ a field of $5 \mathrm{~T}$ was applied and then turned off to give an isothermal remanent magnetization. The samples were then heated to $300 \mathrm{~K}$ while measuring the magnetic moment in intervals of $2 \mathrm{~K}$. The MPMS is equipped with an internal field compensation coil and has a residual field of less than $100 \mu \mathrm{T}$.

Measurements of induced magnetization as a function of temperature or field were also made on a MPMS SQUID magnetometer at the University of Bremen. In the thermal experiments samples were initially cooled to $2 \mathrm{~K}$, either in the absence of a magnetic field (ZFC) or in the presence of a weak $50 \mathrm{mT}$ field (FC). At $2 \mathrm{~K}$ a magnetic field of $50 \mathrm{mT}$ was applied and the sample was then heated to $100 \mathrm{~K}$ with the magnetic moment measured at intervals of approximately $2 \mathrm{~K}$. Sample magnetization was also measured as a function of field (hysteresis loop) at 5, 25, 77 and $300 \mathrm{~K}$, in fields from -7 to $7 \mathrm{~T}$ after cooling the samples in zero field.

\section{Results}

\section{Isothermal remanent magnetization (IRM)}

All IRM acquisition curves show a rapid increase in magnetization in low fields and saturation is reached by $200 \mathrm{mT}$ (Figure 1). The shape of the curve is suggestive of the ferrimagnetic minerals magnetite and/or maghemite $\left(\gamma-\mathrm{Fe}_{2} \mathrm{O}_{3}-\right.$ an oxidation product of magnetite with slightly smaller saturation magnetization). By giving the samples a saturation magnetization in one direction and then acquiring the IRM in the antipodal direction, it is possible to obtain the coercivity of remanence $\left(\mathrm{H}_{\mathrm{cr}}\right)$. The values are 35 and $25 \mathrm{mT}$ for $\mathrm{GH}$ and $\mathrm{NU}$, respectively, and are approximately the same at $77 \mathrm{~K}$ and room temperature. A difference in saturation remanence of $7.1 \times 10^{-7}$ and $3.7 \times 10^{-7}$

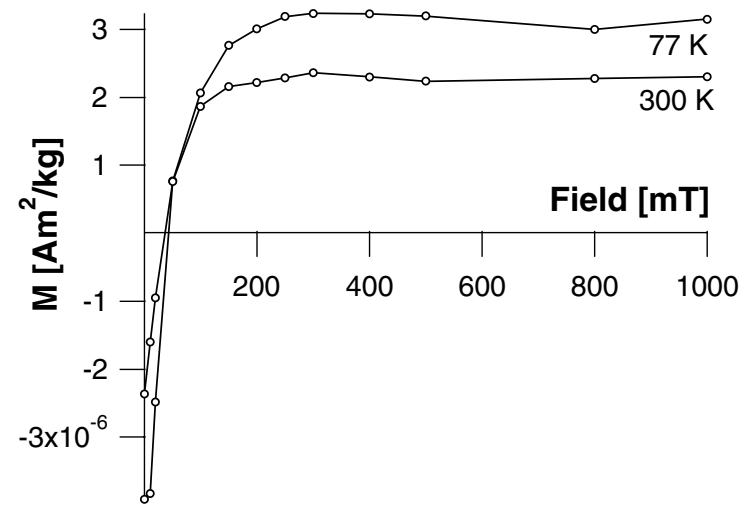

Figure 1. Acquisition of Isothermal Remanent Magnetization (IRM) at 77 and $300 \mathrm{~K}$ for sample GH, showing a low coercivity phase and saturation between 200 and $300 \mathrm{mT}$.

$\mathrm{Am}^{2} / \mathrm{kg}$ between the IRM at $300 \mathrm{~K}$ and the IRM at $77 \mathrm{~K}$ can be seen for $\mathrm{GH}$ and NU, respectively, and is due to ultrafine particles in the sample that are superparamagnetic at $\mathrm{RT}$ but ferrimagnetic at $77 \mathrm{~K}$.

\section{Thermal demagnetization of low temperature isothermal remanent magnetization}

The initial IRM intensities of GH and NU at $2 \mathrm{~K}$ are $4.5 \times 10^{-4}$ and $3.3 \times 10^{-4} \mathrm{Am}^{2} / \mathrm{kg}$, respectively, when cooled in zero field. On heating, there is a sharp decrease in intensity of magnetization between $2 \mathrm{~K}$ and $20 \mathrm{~K}$. The field-cooled samples show a higher initial IRM intensity at $2 \mathrm{~K}$ of $6.4 \times 10^{-4}$ for $\mathrm{GH}$ and $4.6 \times 10^{-4} \mathrm{Am}^{2} / \mathrm{kg}$ for NU. Again, there is a sharp decrease of magnetization on heating between 2 and $30 \mathrm{~K}$. A slight change in slope is seen in the ZFC curve between 10 and $12 \mathrm{~K}$ (Figure 2). For both samples the FC and ZFC curves superimpose indistinguishably at a temperature between 55 and $60 \mathrm{~K}$.

\section{Induced magnetization: DC susceptibility}

The measurement of the induced magnetic moment as a function of temperature in a constant field after ZFC or FC is a useful method in the characterization of horse spleen ferritin (MohieEldin et al. 1994; Makhlouf et al. 1997; Friedman et al. 1997; Gilles et al. 2000). Figure 3 shows the DC susceptibility as a function of temperature for ZFC and FC. Both curves superimpose above the bifurcation point at approximately $60 \mathrm{~K}$. This 


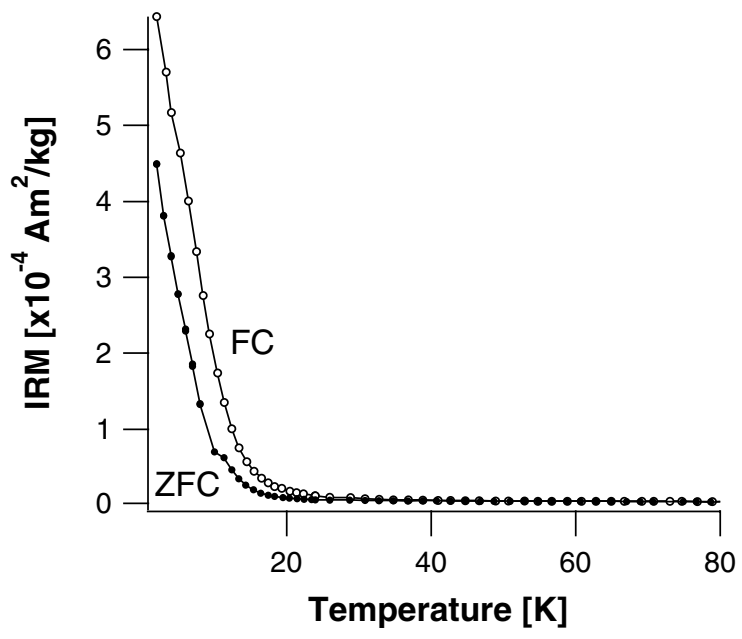

Figure 2. Field cooled (FC) and zero-field cooled (ZFC) thermal demagnetization curves of sample $\mathrm{GH}$.

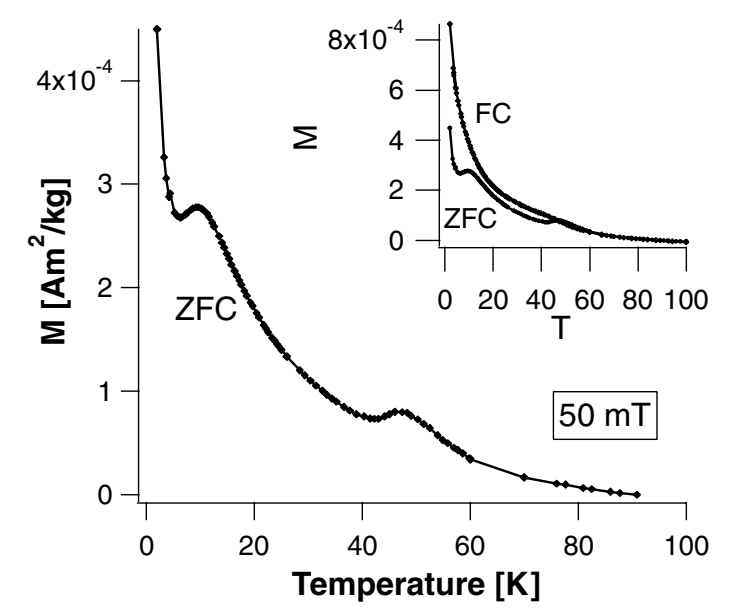

Figure 3. Induced ZFC-magnetization curves as a function of temperature for sample $\mathrm{GH}$ between 2 and $100 \mathrm{~K}$ in an applied field of $50 \mathrm{mT}$. The inset shows the FC- and ZFC curves.

point indicates the maximum blocking temperature of the particles in the sample. The FC curve increases rapidly below this temperature, but does not follow the Curie-Weiss law for paramagnetic behaviour. The ZFC curve exhibits two local maxima at 10 and $48 \mathrm{~K}$. The local maximum around $10 \mathrm{~K}$ is typical for the magnetic ordering of ferritin in both horse spleen (Makhlouf et al. 1997) and the human brain (Dubiel et al. 1999). The second local maximum at $48 \mathrm{~K}$ is typical for pure magnetite (Walz \& Kronmuller 1991; Walz \& Kronmuller 1994; Moskowitz et al. 1998; Muxworthy 1999). There is a very strong increase in the ZFC curve below $6 \mathrm{~K}$, which suggests further blocking of a magnetic phase; this will be discussed below.

\section{Induced magnetization: hysteresis}

The magnetic hysteresis curves are dominated by a strong diamagnetic signal from the tissue also reported by Hautot et al. (2003). The diamagnetic signal can be removed from the total signal by subtracting the fit to the curve at $300 \mathrm{~K}$, which is linearly dependent on temperature. Removal of the linear component will also remove any contribution to the magnetization from paramagnetic materials as they also have a linear, albeit positive response. After the removal of the linear components at $300 \mathrm{~K}$, a weak, closed hysteresis loop remains (Figure 4a). The magnetization is saturated by approximately $200 \mathrm{mT}$, which is typical for magnetite and/or maghemite. The hysteresis at $77 \mathrm{~K}$ is similar to the loop at $300 \mathrm{~K}$, but the intensity of the saturation magnetization increases from $7.7 \times 10^{-5}$ to $7.8 \times 10^{-4} \mathrm{Am}^{2} / \mathrm{kg}$ (Figure 4b). This indicates the further ordering in the low coercivity phase. At $25 \mathrm{~K}$, the hysteresis loop has a contribution from a phase with higher coercivity, which can be attributed to the ordering of ferritin (Figure 4c). The loop is nearly closed, since the average blocking temperature of the ferritin was determined to be $10 \mathrm{~K}$. Therefore, most of the particles in the sample are already in the superparamagnetic state. The lower coercivity phase is still present. The intensity of the magnetization at $7 \mathrm{~T}$ is $13 \times 10^{-3} \mathrm{Am}^{2} / \mathrm{kg}$. At $5 \mathrm{~K}$, the hysteresis loop after ZFC is open and dominated by the ferritin (Figure 5). The magnetization at $7 \mathrm{~T}$ is $26 \times 10^{-3} \mathrm{Am}^{2} / \mathrm{kg}$ and the ordered phase has a coercivity of about $50 \mathrm{mT}$.

\section{Discussion}

Both brain samples reveal clearly the presence of two magnetic compounds. Room temperature measurements and the measurements at $77 \mathrm{~K}$ are dominated by a low coercivity phase, while at low temperature a high coercivity phase is predominant.

The low coercivity phase at room temperature and $77 \mathrm{~K}$ is evident in the results of the IRM measurements and the hysteresis curves and is 

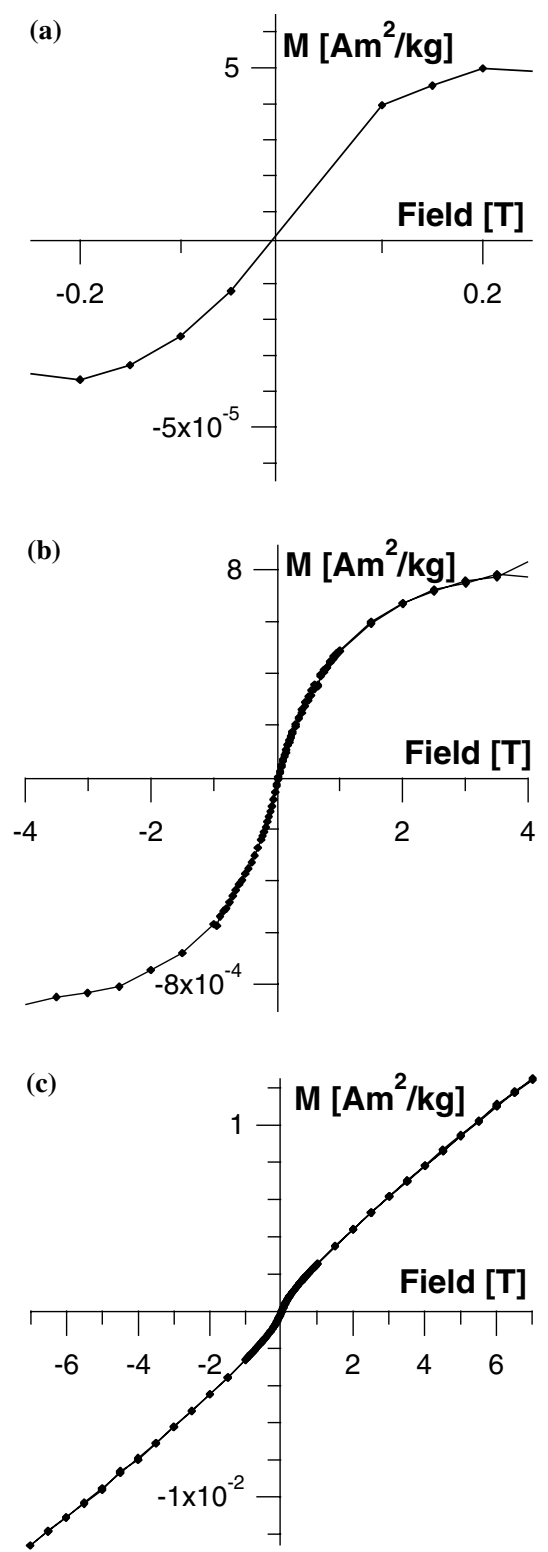

Figure 4. Hysteresis curves from -7 to $7 \mathrm{~T}$ of sample $\mathrm{GH}$ after subtracting the linear components from the curve. (a) Hysteresis at $300 \mathrm{~K}$, (b) hysteresis at $77 \mathrm{~K}$, (c) hysteresis at $25 \mathrm{~K}$ (Note the change in scale).

suggested to be magnetite and/or maghemite. The shape of the IRM curves (Figure 1), typically showing saturation between 200 and $250 \mathrm{mT}$, and the small remanent coercivity $\left(\mathrm{H}_{\mathrm{cr}}\right)$ between 20 and $30 \mathrm{mT}$ are consistent for either of these materials. Induced magnetization measurements (Figure 3) reveal an anomaly around $50 \mathrm{~K}$ that has only been reported for low temperature behaviour in pure

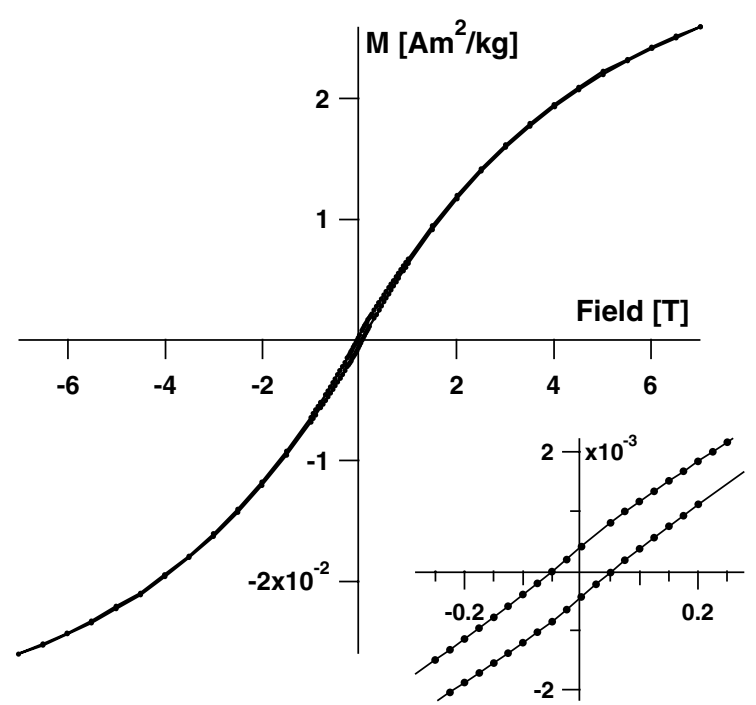

Figure 5. Hysteresis at $5 \mathrm{~K}$ from -7 to $7 \mathrm{~T}$ of sample $\mathrm{GH}$. The inset shows the coercivity field of $50 \mathrm{mT}$.

magnetite (Walz \& Kronmuller 1991; Walz \& Kronmuller 1994; Moskowitz et al. 1998; Muxworthy 1999). Assuming that single-domain magnetite is responsible for the magnetization, an estimate of the magnetite concentration of ordered grains in the tissue can be made from the saturation IRM at $77 \mathrm{~K}$, using a value of $46 \mathrm{Am}^{2} / \mathrm{kg}$ for $\mathbf{J}_{\mathrm{S}} / 2$ (Moskowitz 1993). This value assumes an ensemble of randomly oriented, non-interacting particles. A concentration of 69 and $82 \mathrm{ng} / \mathrm{g}$ can be calculated for samples $\mathrm{GH}$ and NU, respectively. These values are comparable to earlier studies on magnetite concentration in hippocampus tissue from epileptic patients (SchultheissGrassi \& Dobson 1999). It should be noted that the estimated concentration is temperaturedependent due to the presence of superparamagnetic grains. Below $77 \mathrm{~K}$, the high coercivity phase becomes predominant, which can be seen in hysteresis measurements at 25 and $5 \mathrm{~K}$ (Figures $4 \mathrm{c}$ and 5). The hysteresis remains closed at $25 \mathrm{~K}$, even though we see a low coercivity phase in the IRM acquisition curves. This is because the magnetization of the ferritin dominates the induced magnetization at low temperature. The hysteresis at $5 \mathrm{~K}$ shows an $\mathrm{H}_{\mathrm{cr}}$ of $50 \mathrm{mT}$. There is a strong increase in the magnetization intensity of the high coercivity phase with decreasing temperature. Both curves show no saturation at a field of $7 \mathrm{~T}$. FC and ZFC curves from the thermal demagnetization 
measurements decrease rapidly between 2 and $30 \mathrm{~K}$ and join between 55 and $60 \mathrm{~K}$ (Figure 2). For the induced magnetization measurements, the FC and ZFC curves superimpose also at a temperature of $60 \mathrm{~K}$ (Figure 3). The phase shows ordering between 6 and $12 \mathrm{~K}$ that causes the local maximum around $10 \mathrm{~K}$.

Results from all three magnetic methods support ferritin as the high coercivity phase. The increase in the contribution to intensity and the increase in coercivity seen in the hysteresis curves, the local maxima found in the ZFC curves of the induced magnetization and thermal demagnetization and the superposition of the ZFC and FC curves are characteristic for ferritin. Hysteresis measurements are very similar to measurements done for horse spleen ferritin. Perfect antiferromagnets (AFM) do not show hysteresis, therefore the open loops are probably due to the switching of frustrated surface spins at the ferritin core or to a defect moment (Néel 1962). The ordering temperature between 6 and $12 \mathrm{~K}$ is also typical for ferritin; horse spleen ferritin has an average blocking temperature from 10 to $20 \mathrm{~K}$ (Kilcoyne \& Cywinski 1995; Makhlouf et al. 1997; Friedman et al. 1997; Gilles et al. 2000). The only magnetic study on human brain ferritin from globus pallidus by Dubiel et al. (1999) reports an average blocking temperature of $8.5 \mathrm{~K}$. The distribution of particle volume, energy barriers and therefore blocking temperatures is known from many superparamagnetic systems. At the bifurcation point of FC and ZFC curves, the largest particle in the sample starts to be unblocked and the whole sample is superparamagnetic above $60 \mathrm{~K}$. The FC and ZFC curves of both thermal demagnetization and induced magnetization superimpose at this temperature. This is in agreement with the results reported for horse- and human spleen ferritin by Allen et al. (2000).

The strong increase of magnetic moment below $6 \mathrm{~K}$ might be due to a paramagnetic phase in the tissue, such as heme iron from blood in the tissue. Blood is reported to show no irregularity in the ZFC curve; the induced magnetization after FC and ZFC superimpose and show a paramagneticlike decay (Mosiniewicz-Szablewska et al. 2003). Similar behaviour of induced magnetization as a function of temperature has been reported for synthetically formed iron-oxyhydroxide cores inside the protein shell apoferritin using sol-gels
(Rao et al. 2001). An open question is whether the behaviour below $6 \mathrm{~K}$ can be due to (1) a mutated ferritin core, which forms a different iron phase or (2) an additional crystalline or non-crystalline phase within the ferritin core. The influence of blood on the magnetic properties at low temperature will be the focus of a future investigation.

Our measurements show that it is possible to obtain consistent results from different methods that support two magnetic phases, ferritin and magnetite, in tumour tissue from human brain resected from the mesial temporal lobe from MTLE patients.

\section{Acknowledgements}

We thank the Institute for Geosciences at the University of Bremen, especially Dr. Thomas Frederichs and Prof. Dr. Ulrich Bleil for the use of the MPMS and very helpful discussions. All procedures were approved by the University HospitalZurich Medical Ethics Committee. Jon Dobson gratefully acknowledges the support of a Royal Society/Wolfson Foundation Research Merit Award.

\section{References}

Allen PD, St Pierre TG, Chua-anusorn W, Strom V, Rao KV. 2000 Low-frequency low-field magnetic susceptibility of ferritin and hemosiderin. Biochim Biophys Acta-Mol Basis Dis 1500, 186-196.

Beard JL, Connor JR, Jones BC. 1993 Iron in the Brain. Nutr Rev 51, 157-170.

Dobson J. 2001 Nanoscale biogenic iron oxides and neurodegenerative disease. FEBS Lett 496, 1-5.

Dobson J, Grassi P. 1996 Magnetic properties of human hippocampal tissue - evaluation of artefact and contamination sources. Brain Res Bull 39, 255-259.

Dubiel SM, Zablotna-Rypien B, Mackey JB, Williams JM. 1999 Magnetic properties of human liver and brain ferritin. Eur Biophys J Biophys Lett 28, 263-267.

Evans ME, Heller F. Environmental magnetism: principals and applications of enviromagnetics, Amsterdam: Academic press.

Friedman JR, Voskoboynik U, Sarachik MP. 1997 Anomalous magnetic relaxation in ferritin. Phys Rev B 56, 10793-10796.

Fuller M, Dobson J, Wieser HG, Moser S. 1995 On the sensitivity of the human brain to magnetic-fields - evocation of epileptiform activity. Brain Res Bull 36, 155-159.

Gilles C, Bonville P, Rakoto $\mathrm{H}$ et al. 2002 Magnetic hysteresis and superantiferromagnetism in ferritin nanoparticles. J Magn Magn Mater 241, 430-440.

Gilles C, Bonville P, Wong KKW, Mann S. 2000 NonLangevin behaviour of the uncompensated magnetization 
in nanoparticles of artificial ferritin. Eur Phys $J$ B 17, 417427

Hautot D, Pankhurst QA, Khan N, Dobson J. 2003 Preliminary evaluation of nanoscale biogenic magnetite in Alzheimer's disease brain tissue. Proc R Soc Lond Ser B-Biol Sci 270, S62-S64.

Ikeda M. 2001 Iron overload without the C282Y mutation in patients with epilepsy. J Neurol Neurosurg Psychiatry 70, 551-553.

Kilcoyne SH, Cywinski R. 1995 Ferritin - a model superparamagnet. J Magn Magn Mater 140, 1466-1467.

Kirschvink JL, Kobayashi-Kirschvink A, Woodford BJ. 1992 Magnetite biomineralization in the human brain. Proc Natl Acad Sci USA 89, 7683-7687.

Kobayashi AK, Yamamoto N, Kirschvink JL. 1997 Studies of inorganic crystals in biological tissue: magnetite in human tumors. J Jpn Soc Powder Powder metall 44, 294-300.

Luis F, del Barco E, Hernandez JM et al. 1999 Resonant spin tunneling in small antiferromagnetic particles. Phys Rev $B$ 59, 11837-11846.

Maher BA, Thompson, R. 1999 Quaternary climates, environments and magnetism, Cambridge: Cambridge University Press.

Makhlouf SA, Parker FT, Berkowitz AE. 1997 Magnetic hysteresis anomalies in ferritin. Phys Rev B 55, 14717-14720.

Mohie-Eldin MEY, Frankel RB, Gunther L. 1994 A comparison of the magnetic-properties of polysaccharide iron complex (Pic) and ferritin. J Magn Magn Mater 135, 65-81.

Mosiniewicz-Szablewska E, Slawska-Waniewska A, Swiatek K et al. 2003 Electron paramagnetic resonance studies of human liver tissues. Appl Magn Reson 24, 429-435.

Moskowitz BM. 1993 High-temperature magnetostriction of magnetite and titanomagnetites. $J$ Geophys Res-Solid Earth 98, 359-371.

Moskowitz BM, Jackson M, Kissel C. 1998 Low-temperature magnetic behavior of titanomagnetites. Earth Planet Sci Lett $157,141-149$
Muxworthy AR. 1999 Low-temperature susceptibility and hysteresis of magnetite. Earth Planet Sci Lett 169, 51-58.

Néel L. 1962 Proprietes magnetiques des grains fins antiferromagnetiques - superparamagnetisme et superantiferromagnetisme. J Phys Soc Jpn 17, 676.

Quintana C, Lancin M, Marhic C et al. 2000 Initial studies with high resolution TEM and electron energy loss spectroscopy studies of ferritin cores extracted from brains of patients with progressive supranuclear palsy and Alzheimer disease. Cell Mol Biol 46, 807-820.

Rao MS, Dubenko IS, Roy S, Ali N, Dave BC. 2001 Matrixassisted biomimetic assembly of ferritin core analogues in organosilica sol-gels. J Am Chem Soc 123, 1511-1512.

Schafer FQ, Qian SY, Buettner GR. 2000 Iron and free radical oxidations in cell membranes. Cell Mol Biol 46, 657-662.

Schultheiss-Grassi PP, Dobson J. 1999 Magnetic analysis of human brain tissue. Biometals 12, 67-72.

Seehra MS, Babu VS, Manivannan A, Lynn JW. 2000 Neutron scattering and magnetic studies of ferrihydrite nanoparticles. Phys Rev B 61, 3513-3518.

Ueda Y, Willmore LJ. 2000 Sequential changes in glutamate transporter protein levels during $\mathrm{Fe}^{3+}$-induced epileptogenesis. Epilepsy Res 39, 201-209.

Ueda Y, Willmore LJ, Triggs WJ. 1998 Amygdalar injection of $\mathrm{FeCl}_{3}$ causes spontaneous recurrent seizures. Exp Neurol 153, 123-127.

Walz F, Kronmuller H. 1991 Evidence for a single-stage Verweytransition in perfect magnetite. Philos Mag B-Phys Condens Matter Stat Mech Electron Opt Magn Prop 64, 623-628.

Walz F, Kronmuller H. 1994 Analysis of magnetic point-defect relaxations in electron-irradiated magnetite. Phys Status Solidi B-Basic Res 181, 485-498.

Zhao GH, Bou-Abdallah F, Arosio P et al. 2003 Multiple pathways for mineral core formation in mammalian apoferritin. The role of hydrogen peroxide. Biochemistry 42, 3142-3150. 